\title{
The Holomorphic Equivalence Problem for a Class of Reinhardt Domains
}

\author{
By \\ Isao NARUKI
}

\section{Introduction}

The holomorphic equivalence problem for domains in $\boldsymbol{C}^{n}$ is very difficult and up to now one who has studied this problem has been forced to take one of the following two viewpoints to it. The older one taken by many of complex analysts discussed mainly certain special kinds of domains in $\boldsymbol{C}^{2}$ in order to obtain rather complete solutions by case-by-case arguments. See for example [11], [9], [4], [5], [12]. But without any essential modifications such methods seem to cause difficulties when one tries to generalize them to the case $n>2$. The other, rather new viewpoint which was initiated by E. Cartan has only aimed at the investigation of bounded homogeneous domains in $\boldsymbol{C}^{n}$ and in this case the main tool is Lie group theory, of course. See [3], [2], [8], [6], [7], [10].

But very little was done yet concerning the application of Lie group theory to the equivalence problem for inhomogeneous domains (even for Reinhardt domains). So this paper attempts, from the group-theoretic point of view, to investigate the equivalence problem for a special kind of Reinhardt domains in $\boldsymbol{C}^{n}$, as was considered by Thullen [12] when $n=2$. The most difficult part of this problem will be solved by Theorem A, which holds for general Reinhardt domains and is also interesting in its own right. Holomorphic automorphism groups of such domains are also explicitly determined. See Theorem B. Unfortunately such simple domains are not adequate to solve the equivalence problem for general Reinhardt 
domains except for the two dimensional case, as will be seen from Theorem $B^{\text {bis. }}$. Nevertheless the author hopes that the method of this paper would be a first step toward the general equivalence problem for Reinhardt domains.

The author wishes to express his sincere thanks to Professor M. Ise for suggesting this problem, and also to N. Tanaka for many valuable advices.

\section{Anouncement of Results}

Let $D$ be a bounded domain ${ }^{1)}$ in $C^{n}$ and $A(D)$ be the group of its holomorphic automorphisms. The identity component of $A(D)$ is denoted by $A^{0}(D)$. (Recall that $A(D)$ is always a Lie transformation group of $D$ with respect to the compact open topology.) A bounded domain $D$ is called a Reinhardt domain if it is always mapped onto itself by any of the following transformations :

$$
T\left(\xi^{1}, \cdots, \xi^{n}\right) z=\left(e^{i \xi^{1}} z_{1}, \cdots, e^{i \xi^{n}} z_{n}\right)
$$

where, $\xi^{1}, \cdots, \xi^{n}$ are real numbers. The group of such transformations is denoted by $T$ and the restriction of $T$ to a Reinhardt domain is also denoted by the same letter as far as confusion may not occur. Note that $T$ has the origin as its unique fixed point and that $T$ operates freely on $D^{*}$ for a Reinhardt domain $D$ where we set $D^{*}=\left[z \in D ; z_{1} \neq 0, \cdots, z_{n} \neq 0\right]$. These facts suggest us the importance of the orbit $O_{D}$ of $A^{0}(D)$ passing through the origin and the importance of the mutual relation of $O_{D}$ and $D^{*}$. In fact we have the following theorem which clarifies these matters.

Theorem A. Let $D$ be a bounded Reinhardt domain in $\boldsymbol{C}^{n}$ and assume that $O_{D}-D^{*}=(0)$, then $O_{D}=(0)$.

When $n=2$, this is due to Thullen [2]. His proof has some ambiguous points. Nevertheless the proof of this paper was suggested by his, and will be carried essentially along the same line, though it requires Cartan-Malcev-Iwasawa's theorem in the theory of Lie groups.

1) Domains considered in this paper contain the origin unless otherwise stated. 
We shall now talk of the purpose of this paper. Let $D_{a}$ be the Reinhardt domain in $\boldsymbol{C}^{n}$ defined by $\sum_{j=1}\left|z_{j}\right|^{\alpha}{ }_{j}<1, \alpha=\left(\alpha_{1}, \cdots, \alpha_{n}\right) \alpha_{j}>0$.

Problem. Determine $A\left(D_{\alpha}\right) !$ When are $D_{\alpha}, D_{\alpha^{\prime}}$ mutually holomorphically equivalent?

Theorem A gives us a partial answer to this. In fact, one sees easily by Theorem A that $A^{0}\left(D_{x}\right)=T$ when $\alpha_{j} \neq 2(1 \leq j \leq n)$, and that $D_{\alpha}, D_{\alpha^{\prime}}$ are mutually equivalent if and only if $\alpha=\alpha^{\prime}$ provided that $\alpha_{j} \neq 2, \alpha_{j}^{\prime} \neq 2$ for $1 \leq j \leq n$. Theorem $\mathrm{A}$ and this last fact will be proved in Section 1.

For the case $\alpha_{j}=2$ for some $j$, the infinitesimal group $\mathfrak{A}\left(D_{a}\right)$ of $A^{0}\left(D_{\alpha}\right)$ can be determined heuristically by fairly elementary argument. Although this is mere digression we include this in Section 2. Section 2 also includes some results on the structure of $\mathfrak{A}\left(D_{\alpha}\right)$ which will be important in Section 3 .

In Section 3 we justify the result conjectured in Section 2 by the induction on the number of independent variables. In summary we obtain

Theorem B. Under previous notations $\mathfrak{A}\left(D_{\alpha}\right)$ consists of all vecter fields of the following form:

$$
\operatorname{Re}\left(\sum_{j=1}^{r} \frac{2}{\alpha_{j}}\left(i c_{j}-\sum_{k=r+1}^{n} \bar{c}_{k} z_{k}\right) z_{j} \frac{\partial}{\partial z_{j}}+\sum_{j=r+1}^{n}\left(c_{j}+\sum_{k=r+1}^{n} c_{j k} z_{k}-\left(\sum_{k=r+1}^{n} \bar{c}_{k} z_{k}\right) z_{j} \frac{\partial}{\partial z_{j}}\right)\right.
$$

where $c_{1}, \cdots, c_{r}$ are real, $c_{r+1}, \cdots, c_{n}$ are complex, $\left(c_{j k}\right)$ is anti-Hermitian, $\alpha_{1} \neq 2, \cdots, \alpha_{r} \neq 2, \alpha_{r+1}=\cdots=\alpha_{n}=2$. Moreover $D_{\alpha}, D_{\alpha^{\prime}}$ are mutually equivalent if and only if $\alpha=\alpha^{\prime}$ provided that $\alpha_{1} \geq \alpha_{2} \geq \cdots \geq \alpha_{n}, \alpha_{1}^{\prime} \geq$ $\alpha_{2}^{\prime} \geq \cdots \geq \alpha_{n}^{\prime}$.

We also indicate here that the method presented in Section 2-3 is applicable to the equivalence problem for a more wider class of domains as defined below. For $\alpha=\left(\alpha_{1}, \cdots, \alpha_{s}\right)$ and $I=\left(i_{1}, \cdots, i_{s}\right)$ (where $\alpha_{j}>0(1 \leq j \leq s), i_{p}(1 \leq p \leq s)$ : positive integers) we define the domain as follows:

$$
D_{\alpha, I}: \sum_{p=1}^{s}\left(\sum_{j=1}^{i p}\left|z_{j p}\right|^{2}\right)^{\alpha} p<1
$$

where $\left(z_{11}, \cdots, z_{i_{1} 1}, z_{12}, \cdots, z_{i_{2} 2}, \cdots, z_{1 s}, \cdots, z_{i_{s s}}\right)$ is the coordinate of 
$\boldsymbol{C}^{n}\left(n=\sum_{p=1}^{s} i_{p}\right)$. Without loss of generality we may assume that $\alpha_{1} \neq 1, \cdots, \alpha_{s-1} \neq 1$. Then we have the following theorem

Theorem $\mathbf{B}^{\text {bis. }} \mathfrak{A}\left(D_{a, I}\right)$ consists of vector fields of the following form :

$$
\begin{array}{r}
\operatorname{Re}\left(\sum_{p=1}^{s-1} \alpha_{p}^{-1} \sum_{j=1}^{i_{p}}\left(\sum_{k=1}^{i_{p}} c_{j k}^{p} z_{k p}-\left(\sum_{k=1}^{i_{s}} \bar{c}_{k} z_{k s}\right) z_{j p}\right) \frac{\partial}{\partial z_{j p}}\right. \\
\left.+\sum_{j=1}^{i_{s}}\left(c_{j}+\sum_{k=1}^{i_{s}} c_{j k}^{s} z_{k s}-\left(\sum_{k=1}^{i_{s}} \bar{c}_{k} z_{k s}\right) z_{j s}\right) \frac{\partial}{\partial z_{j s}}\right)
\end{array}
$$

if $\alpha_{s}=1$,

$$
\operatorname{Re}\left(\sum_{p=1}^{s} \alpha_{p}^{-1} \sum_{j=1}^{i p} \sum_{k=1}^{i p} c_{j k}^{n} z_{k p} \frac{\partial}{\partial z_{j p}}\right)
$$

if $\alpha_{s} \neq 1$ where $C^{p}=\left(c_{j k}^{p}\right)$ are anti-Hermitian matrices.

$D_{\alpha, I}, D_{\alpha^{\prime}, I^{\prime}}$ are mutually equivalent if and only if $\alpha=\alpha^{\prime}, I=I^{\prime}$ provided that $\alpha_{1} \geq \cdots \geq \alpha_{s}, \alpha_{1}^{\prime} \geq \cdots \geq \alpha_{s}{ }^{\prime}$.

The proof of this is quite similar to that of Theorem $\mathrm{B}$, and causes mere inessential complications, therefore shall be omitted.

\section{Section 1}

As stated before we shall prove Theorem A in this Section. Most of the notations introduced before shall be preserved here. Before proceeding we shall introduce some notations. Let $\boldsymbol{R}_{+}^{n}$ denote $\left[\left(x_{1}, \cdots, x_{n}\right): x_{I}>0, x_{2}>0, \cdots, x_{n}>0\right]$ and let $\overline{\boldsymbol{R}_{+}^{n}}$ be $\left[\left(x_{1}, \cdots, x_{n}\right)\right.$ : $\left.x_{1} \geq 0, x_{2} \geq 0, \cdots, x_{n} \geq 0\right]$. Define a mapping of $\boldsymbol{C}^{n}$ onto $\overline{\boldsymbol{R}_{+}^{n}}$ by setting :

$$
\pi\left(z_{1}, \cdots, z_{n}\right)=\left(\left|z_{1}\right|, \cdots,\left|z_{n}\right|\right) .
$$

When $K$ is a subset of $\boldsymbol{R}_{+}^{n}, \hat{K}$ denotes $\underset{y \in K}{\cup}\left[\left(x_{1}, \cdots, x_{n}\right): x_{1} \leq y_{1}, \cdots\right.$, $\left.x_{n} \leq y_{n}\right]$.

Proposition 1.1. Let $K$ be a arcwise-connected subset of $\overline{\boldsymbol{R}_{+}^{n}}$ such that $K-R_{+}^{n}=(0)$. Then every function which is holomorphic on a neibourhood of $\pi^{-1}(K)$ has the unique holomorphic extension to a neibourhood of $\pi^{-1}(\hat{K})$.

The usual proof of this depends on deformation of a cycle to which Cauchy integral formula is applied and is fairly standard, 
shall be therefore neglected.

Now let $D$ be a bounded Reinhardt domain such that $O_{D}-D^{*}$ $=(0)$, which we fix in the whole Section. For brevity we set $G=A^{0}(D)$ and also set $S^{*}=S \cap D^{*}$ for every subset $S$ of $D$. As usual $O_{D}$ is naturally identified with the left coset space $G / I$ where $I$ is the isotropy subgroup of $G$ at the origin which is automatically compact. Under this identification $H / I$ is a complete inverse image of $\pi$ when $H$ is a Lie subgroup of $G$ containing $I . \quad T$ acts freely on $(H / I)^{*}$ and under this action $(H / I)^{*}$ is a (trivial) principal fiber bundle over $\pi\left((H / I)^{*}\right)$ with structure group $T$. Further $\pi\left((H / I)^{*}\right)$ is $C^{\infty}$ manifold of $\boldsymbol{R}_{+}^{n}$ (which is not necessarily a topological subspace).

Assertion 1. 1. If $(H / I)^{*}$ is non-empty, then $\pi\left((H / I)^{*}\right)$ is a 1-dimensional submanifold of $\boldsymbol{R}_{+}^{*}$.

Proof. Define a holomorphic function $f$ setting $f(z)=z_{1}$, and let $h \in H$. Then it follows from $O_{D}-D^{*}=(0)$ that the intersection of $O_{D}^{*}$ and the analytic surface $f \cdot h^{-1}=0$ contains only one point $h \cdot O$ $(o$; the origin). In particular it holds

$$
H / I \cap\left[z \in D: f \cdot h^{-1}(z)=0\right]=(h \cdot o) .
$$

Now let $K$ be $\pi(H / I)$ and notice that $K$ satisfies the hypothesis of Proposition 1.1. Draw a curve $l$ from $o$ in $K$ such that $l \cap \boldsymbol{R}_{+}^{n}$ is non-empty and set $S=\pi^{-1}(\hat{l}) \cap H / I$. Then we conclude $S=\pi^{-1}(l)$. For, suppose in contrary $h \cdot o \in S-\pi^{-1}(l)$ where $h$ is an element of $H$. Then it follows from $(*)$ that $1 / f \cdot h^{-1}$ is holomorphic on $\pi^{-1}(l)$, and hence $1 / f \cdot h^{-1}$ can be holomorphically extendable to a neibourhood of $\pi^{-1}(\hat{l})$, but this contradicts to $f \cdot h^{-1}(h \cdot o)=0$, where $h \cdot o \in \pi^{-1}(\hat{l})$. Thus $S=\pi^{-1}(l)$, which implies $\operatorname{dim} \pi\left((H / I)^{*}\right)=1$ since $\pi(S)$ contains an open subset of $\pi\left((H / I)^{*}\right)$. Assertion 1. is proved.

It follows in particular that $\pi\left(O_{D}^{*}\right)$ is 1-dimensional. If we take a maximal compact subgroup containing $I$ as $H$, Assertion 1 shows that $\pi\left((H / I)^{*}\right)$ a closed and open submanifold of $\pi\left(O_{D}^{*}\right)$. Since both $O_{D}^{*}$ and $(H / I)^{*}$ are complete inverse image of $\pi$, it follows either that $I$ is a maximal compact subgroup of $G$, or that $G$ is compact. 
But the latter case must be excluded unless $G=I$. For suppose that $G$ is compact, and that $O_{D}^{*} \neq \phi$, then $\pi\left(O_{D}^{*}\right)$ is 1-dimensional and $\pi\left(O_{D}^{*}\right) \cup(0)$ is compact. Let $\psi$ be a diffeomorphism of $\boldsymbol{R}$ onto $\pi\left(O_{D}^{*}\right)$. Set $l=\psi([t \in R: t \leq o])$. Then, since $\pi\left(O_{D}^{*}\right) \cup(0)$ is compact, $\hat{l}$ contains $\psi([t \in \boldsymbol{R}: t>a])$ for sufficiently large $a$. Quite similarly to the proof of Assertion 1, we deduce that $l$ must necessarily contain $\psi([t: t>a])$, and this is a contradiction. Thus, at any rate, we have

Assertion 1.2. I is a maximal compact subgroup of $G$.

Now theorem A can be easily proved as follows. Suppose that $(G / I)^{*} \neq \phi$, it has the same homotopy type as n-dimensional sphere since $G / I$ is diffeomorphic to an Euclidean space by Cartan-MalcevIwasawa theorem. While $(G / I)^{*}$ is a principal fiber bundle with fiber $T$ over $\pi\left(O_{D}^{*}\right)$ which is diffeomorphic to $\boldsymbol{R}$, and the homotopy type of $(G / I)^{*}$ must be same as $T$. Contradiction! Thus $G=I$, which completes the proof of Theorem A.

Corollary A. $A^{0}\left(D_{\alpha}\right)=T$ and $D_{a}, D_{\alpha^{\prime}}$ are holomorphically equivalent if and only if $\alpha=\alpha^{\prime}$ provided that $\alpha_{1} \geq \cdots \geq \alpha_{n}, \alpha_{1}{ }^{\prime} \geq \cdots \geq \alpha_{n}{ }^{\prime}$, $a_{j} \neq 2, \alpha_{j}^{\prime} \neq 2(1 \leq j \leq n)$.

In order to prove this, we need the following Lemma.

Lemma 1.2. Let $D, D^{\prime}$ be bounded circular domains in $\boldsymbol{C}^{n}$. Then any holomorphic isomorphism of $D$ onto $D^{\prime}$ which leaves the origin fixed is linear.

This is due to Behnke and H. Cartan (See [1] Satz 64). Now we return to the proof of Corollary A. Recall that $D_{a}$ is defined by the inequality $\sum\left|z_{j}\right|^{a_{j}}<1$. Firstly note that the former half of this Corollary implies the latter half. For, if $A^{0}\left(D_{a}\right)=T$, every element of $A\left(D_{a}\right)$ also fixes the origin since $A^{0}\left(D_{\alpha}\right)$ is a normal subgroup of $A\left(D_{a}\right)$. Thus the origin is intrinsically characterized as the unique fixed point of $A\left(D_{\alpha}\right)$. Therefore every holomorphic isomorphism of $D_{a}$ onto $D_{a^{\prime}}$ must necessarily maps the origin into itself, and hence it must be linear by Lemma 1.2. Similarly the analytic planes $z_{j}=0(1 \leq j \leq n)$ are only $2 n-2$ dimensional orbits of 
$A\left(D_{a}\right)$, so one of these must be mapped into another of these by holomorphic isomorphisms of $D_{\alpha}$ onto $D_{\alpha^{\prime}}$. Thus these isomorphisms are almost trivial ones, and the second half of Corollary A follows immediately.

To show $A^{0}\left(D_{a}\right)=T$, we proceed by the induction on the number of independent variables. Actually this follows immediately from the result of Thullen [2] when $n=2$.

Now assume that $n>2$, and that $A\left(D_{a}\right) \neq I\left(D_{a}\right)$. (Hereafter we denote the isotropy subgroup of $A(D)$ at the origin by $I(D)$.) It follows from Theorem A that $O_{D_{\alpha}}$ contains a point $z^{0} \neq 0$ with $z_{j}^{0}=0$ for some $j$. Let $f$ be an element of $A\left(D_{\alpha}\right)$ which sends $z^{0}$ to the origin, and set $T_{j}(\xi)=T(0, \cdots, \stackrel{j-\text { th }}{\xi}, \cdots, 0)$. Then the 1 -parameter subgroup $f \cdot T_{j}(\zeta) \cdot f^{-1}$ leaves the origin fixed, hence this is a subgroup of $T$. (For, the result of the next section shows that $I\left(D_{a}\right)$ has $T$ as its identity component.) Therefore $f \cdot T_{j}(\xi) \cdot f^{-1}$ coincides with $T_{k}( \pm \xi)$ for some $k,{ }^{2)}$ hence $f$ maps the plane $z_{j}=0$ to the plane $z_{k}=0$. But our inductive hypothesis applied to these planes asserts that $f$ necessarily maps the origin into itself, which contradicts to $z^{0} \neq 0$. Thus $A^{0}\left(D_{\alpha}\right)=T$ and the proof completes.

\section{Section 2}

What is aimed at in this Section is to obtain a conjecture on how the situation will be when the restriction $\alpha_{j} \neq 2$ is dropped. The case we are really concerned about is the following. Suppose that $\alpha_{1}, \cdots, \alpha_{n}$ are all positive even integers. In this case $\partial D_{a}$ is a smooth hypersurface in $\boldsymbol{C}^{n}$. So we can consider the differentiability up to the boundary of $D_{\alpha}$, i.e. we can define $C^{\infty}\left(\bar{D}_{w}\right)$, Diff $\left(\bar{D}_{a}\right)$ as usual. Set

$$
A\left(\bar{D}_{a}\right)=\left[f \in \operatorname{Diff}\left(\bar{D}_{a}\right):\left.f\right|_{D_{a}} \in A^{\circ}\left(D_{a}\right)\right]
$$

and let $\mathfrak{A}\left(\bar{D}_{\alpha}\right)$ denote the infinitesimal group of $A\left(\bar{D}_{\alpha}\right)$. (Here the infinitesimal group of $A\left(\bar{D}_{a}\right)$ means the Lie algebra of generators

2) Note that a 1-parameter subgroup of $T$ leaving an analytic surface fixed must be $T_{R}( \pm \xi)$ for some $k$. 
of all 1-parameter subgroups of $A\left(\bar{D}_{a}\right)$, of course.) Clearly $A\left(\bar{D}_{a}\right)$ consists of all vecter fields of the following form

$$
\operatorname{Re}\left(\sum_{j=1}^{n} f_{j}(z) \frac{\partial}{\partial z_{j}}\right) \text { such that }\left.\operatorname{Re}\left(\sum_{j=1}^{n} \alpha_{j} \bar{z}_{j}\left|z_{j}\right|^{\alpha_{j}-2} f_{j}(z)\right)\right|_{\partial D_{\infty}}=0
$$

where $f_{j}(z) \in C^{\infty}\left(\bar{D}_{\alpha}\right)$ are holomorphic in $D_{\alpha}$.

The last condition means that $\operatorname{Re}\left(\sum_{j=1}^{n} f_{j}(z) \frac{\partial}{\partial z_{j}}\right)$ is tangent to $\partial D_{\alpha}$. Let us now determine $A\left(\bar{D}_{a}\right)$ by solving the above boundary value problem. In order to proceed we require some Lemmas.

Lemma 2.1. Suppose that elements $f_{1}(z), \cdots, f_{n}(z)$ of $C^{\infty}\left(\bar{D}_{a}\right)$ are holomorphic in $D_{\alpha}$, and assume that

$$
\left.\sum_{j=1}^{n} \alpha_{j}\left|z_{j}\right|^{\alpha_{j}-2} z_{j} f_{j}(z)\right|_{\partial D_{\alpha}}=0
$$

Then $f_{1}(z)=f_{2}(z)=\cdots=f_{n}(z)=0$.

Proof. Set $X=\sum_{j} f_{j}(z) \frac{\partial}{\partial z_{j}}$. Then the hypothesis of Lemma 2.1 implies that both $X, \bar{X}$ are tangent to $\partial D_{a}$. In other word $X \psi=$ $\bar{X} \psi=0$ where we put $\psi=\sum_{j}\left|z_{j}\right|^{\alpha_{j}}-1$. Further $[X, \bar{X}]=0$ since $f_{j}$ are holomorphic. Therefore

$\left.\langle d \bar{\partial} \psi \mid X \wedge \bar{X}\rangle\right|_{\partial D_{\alpha}}=\left.X\langle\bar{\partial} \psi \mid \bar{X}\rangle\right|_{\partial D_{\alpha}}-\left.\bar{X}\langle\bar{\partial} \psi \mid X\rangle\right|_{\partial D_{\alpha}}-\left.[X, \bar{X}] \psi\right|_{\partial D_{\alpha}}=0$ and hence

$$
\left.\sum_{j} \alpha_{j}\left|z_{j}\right|{ }^{\alpha_{j}-2}\left|f_{j}(z)\right|^{2}\right|_{\partial D_{\infty}}=\left.\langle\partial \bar{\partial} \psi \mid X \wedge \bar{X}\rangle\right|_{\partial D_{\infty}}=0 .
$$

This proves $f_{j}(z)=0(j=1,2, \cdots, n)$.

Lemma 2.2. If $\alpha_{1}=\cdots=\alpha_{n}=2, \mathfrak{A}\left(\bar{D}_{\alpha}\right)$ consists of vecter fields of the following form:

$$
\operatorname{Re}\left(\sum_{j}\left(c_{j}+\sum_{k} c_{j k} z_{k}-\left(\sum_{k} \bar{c}_{k} z_{k}\right) z_{j}\right) \frac{\partial}{\partial z_{j}}\right)
$$

where $c_{1}, \cdots, c_{n}$ are complex numbers, $C=\left(c_{j k}\right)$ is an anti-Hermitian matrix.

This Lemma is an immediate consequence from the well known fact that pseudo-conformal automorphisms of hypersphere in $\boldsymbol{C}^{n}$ are projective transformations considering $\boldsymbol{C}^{n}$ as imbedded naturally in $P\left(\boldsymbol{C}^{n}\right)$, but we prefer to give a direct proof. 
Proof. Suppose that elements $f_{1}(z), \cdots, f_{n}(z)$ of $C^{\infty}\left(\bar{D}_{a}\right)$ are holomorphic in $D_{a}$ and that they satisfy the boundary condition; $(* *)_{1}$ $\left.\operatorname{Re}\left(\sum_{j} z_{j} f_{j}(z)\right)\right|_{|z|=1}=0$.

For a $\boldsymbol{C}^{n}-(0)$ and $|\zeta|<|a|^{-1}$, we set

$$
F(a, \zeta)=\sum_{j} \bar{a}_{j} f_{j}\left(\zeta a_{1}, \cdots, \zeta a_{n}\right)
$$

Then $(* *)_{1}$ may be written as below :

$$
\left.\operatorname{Re}(\bar{\zeta} F(a, \zeta))\right|_{|\zeta|=|a|^{-1}}=0 .
$$

Adding to this the obvious identity $\operatorname{Re}(\zeta F(a, 0)-\bar{\zeta} F(a, 0))=0$, we obtain

$$
\left.\operatorname{Re}(\bar{\zeta}(F(a, \zeta)-F(a, 0))+\zeta \overline{F(a, 0)})\right|_{|\zeta|=|a|^{-1}}=0
$$

Substituting $\zeta$ by $\frac{|a|^{-2}}{\zeta}$ this, we have

$$
(* *)_{2} \quad \operatorname{Re}\left(\left.|a|^{-2}\left(\frac{F(a, \zeta)-F(a, 0)}{\zeta}+\zeta \overline{F(a, 0)}\right)\right|_{|\zeta|=|a|^{-1}}=0 .\right.
$$

Observe that the terms in the largest parenthesis are regular in the disk $|\zeta|<|a|^{-1}$. Thus we have

$$
(* *)_{3} \quad|a|^{-2}\left(\frac{F(a, \zeta)-F(a, 0)}{\zeta}\right)+\zeta \overline{F(a, 0)}=i C(a)
$$

where $C(a)$ is certain real function of $a$.

So $\frac{\partial F}{\partial \zeta}(a, 0)=i C(a)|a|^{-2}$, whence $\left(c_{j k}\right)$ must be anti-Hermitian if we set $c_{j k}=\frac{\partial f_{j}}{\partial z_{k}}(0)$.

Setting $\zeta=1$ in $(* *)_{3}$ for a such that $|a|<1$, we have

$$
\sum_{j} \bar{a}_{j} f_{j}(a)=\sum_{j} \bar{a}_{j}\left(c_{j}+\sum_{k} c_{j k} a_{k}-\left(\sum_{k} \bar{c}_{k} a_{k}\right) a_{i}\right)
$$

where we set $c_{j}=f_{j}(0)$.

Finally applying $\frac{\partial}{\partial \bar{a}_{j}}$ to the both sides of this, we obtain the desired result.

Now we are ready to determine $\mathfrak{A}\left(\bar{D}_{c}\right)$.

Proposition 2.3. If $\alpha_{j} \equiv 0$ (mod. 2) and if $\alpha_{1} \neq 2, \cdots, \alpha_{r} \neq 2$, $\alpha_{r \mid 1}=\cdots=\alpha_{n}=2(0 \leq r \leq n), \mathfrak{U}\left(\bar{D}_{a}\right)$ consists of all vector fields of the following form: 


$$
\operatorname{Re}\left(\sum_{j=1}^{r} \frac{2}{\alpha_{j}}\left(i c_{j}-\sum_{k=r+1}^{n} \bar{c}_{k} z_{k}\right) z_{j} \frac{\partial}{\partial z_{j}}+\sum_{j=r+1}^{n}\left(c_{j}+\sum_{k=r-1}^{n} c_{j k} z_{k}-\left(\sum_{k=r+1}^{n} \bar{c}_{k} z_{k}\right) z_{j}\right) \frac{\partial}{\partial z_{j}}\right)
$$

where $c_{1}, \cdots, c_{r}$ are real, $c_{r+1}, \cdots, c_{n}$ are complex, $C=\left(c_{j k}\right)$ is antiHermitian.

Proof. If $r=0$, this is just Lemma 2.2. So we prove by the induction on $r$. Assume $r>0$. For brevity we set $z^{\prime}=\left(z_{2}, \cdots, z_{n}\right)$ in this proof. (So for example $f\left(z_{1}, z^{\prime}\right)$ means $f(z) \quad\left(z=\left(z_{1}, z^{\prime}\right)\right.$.) Supppose that $f_{1}, \cdots, f_{n} \in C^{\infty}\left(\bar{D}_{c}\right)$ are holomorphic in $D_{a}$ and that they satisfy

$$
\left.\operatorname{Re}\left(\sum_{j} \alpha_{j}\left|z_{j}\right|^{a_{j}-2} \bar{z}_{j} f_{j}(z)\right)\right|_{\left|z_{1}\right|^{\alpha_{1}=\psi\left(z^{\prime}\right)}}=0
$$

where $\psi\left(z^{\prime}\right)=1-\sum_{j>1}\left|z_{j}\right|^{n_{j}}$. Adding to this the obvious identity $\operatorname{Re}\left(\alpha_{1}\left|z_{1}\right|^{\alpha_{1}-2}\left(z_{1} \overline{f_{1}\left(0, z^{\prime}\right)}-\bar{z}_{1} f_{1}\left(0, z^{\prime}\right)\right)=0\right.$, and using $\left|z_{1}\right|^{\alpha_{1}}=\psi\left(z^{\prime}\right)$, we obtain as before

$$
\begin{gathered}
\operatorname{Re}\left(\alpha_{1} \psi\left(z^{1}\right)\left(\frac{f_{1}\left(z_{1}, z^{\prime}\right)-f_{1}\left(0, z^{\prime}\right)}{z_{1}}\right)+\alpha_{1} \psi\left(z^{\prime}\right)^{1-\left(2 / \alpha_{1}\right)} z_{1} \overline{f_{1}\left(0, z^{\prime}\right)}\right. \\
\left.+\sum_{j>1} \alpha_{j}|z|^{\omega_{j}-1} \bar{z}_{j} f_{j}(z)\right)\left.\right|_{\mid z_{1}{ }^{\prime} \alpha_{1=\psi\left(z^{\prime}\right)}}=0 .
\end{gathered}
$$

Notice that the terms in the largest parenthesis are regular in $z_{1}$ in the disk $\left|z_{1}\right|^{a_{1}}<\psi\left(z^{\prime}\right)$. Hence we have

$$
\begin{gathered}
(* *)_{4} \quad \alpha_{1} \psi\left(z^{\prime}\right)\left(\frac{f_{1}\left(z_{1}, z^{\prime}\right)-f_{1}\left(0, z^{\prime}\right)}{z_{1}}\right)+\alpha_{1} \psi\left(z^{\prime}\right)^{1-\left(2 / \alpha_{1}\right)} z_{1} \overline{f_{1}\left(0, z^{\prime}\right)} \\
\left.+\sum_{j>1} \alpha_{j}\left|z_{j}\right|^{a_{j}-1} \bar{z}_{j} f_{j}(z)\right)=i C\left(z^{\prime}\right)
\end{gathered}
$$

where $C\left(z^{\prime}\right)$ is a real function of $z^{\prime}$. Differentiating this twice with respect to $z_{1}$, we have

$$
\alpha_{1} \psi\left(z^{\prime}\right) \frac{\partial^{2} \widetilde{f}_{1}}{\partial z_{1}^{2}}+\sum_{j>1} \alpha_{j}|z|^{n_{j}-2} \bar{z}_{j} \frac{\partial^{2} f_{j}}{\partial z_{1}^{2}}=0
$$

Or, equivalently

$$
\alpha_{1} \frac{\partial^{2} \bar{f}_{1}}{\partial z_{1}{ }^{2}}=-\sum_{j>1}\left|z_{j}\right|^{\omega_{j}-2} \bar{z}_{j}\left(\alpha_{j} \frac{\partial^{2} f_{j}}{\partial z_{1}{ }^{2}}(z)-\alpha_{1} z_{j} \frac{\partial^{2} \tilde{f}_{1}}{\partial z_{1}{ }^{2}}\right)
$$

where we have put $\widetilde{f}_{1}(z)=\frac{f_{1}\left(z_{1}, z^{\prime}\right)-f_{1}\left(0, z^{\prime}\right)}{z_{1}}$. Notice that the left hand side is holomorphic in $z=\left(z_{1}, \cdots, z_{n}\right)$ while the right hand side 
is not holomorphic unless it vanishes identically. Hence

$$
\frac{\partial^{2} \widetilde{f}_{1}}{\partial z_{1}{ }^{2}}=\frac{\partial^{2} f_{2}}{\partial z_{1}{ }^{2}}=\cdots=\frac{\partial^{2} f_{n}}{\partial z_{1}^{2}}=0 .
$$

Thus we may set

$f_{1}(z)=f_{1}^{0}\left(z^{\prime}\right)+f_{1}^{1}\left(z^{\prime}\right) z_{1}+f_{1}{ }^{2}\left(z^{\prime}\right) z_{1}{ }^{2}, \quad f_{j}(z)=f_{j}{ }^{0}\left(z^{\prime}\right)+f_{j}{ }^{1}\left(z^{\prime}\right) z_{1} \quad(2 \leq j \leq n)$ where $f_{j}{ }^{0}, f_{j}{ }^{1}(1 \leq j \leq n), f_{1}{ }^{2}$ are defined on $\psi\left(z^{\prime}\right) \geq 0$ and are holomorphic in $\psi\left(z^{\prime}\right)>0$. Substituting these into $(* *)_{4}$, we obtain

$(* *)_{5} \quad \alpha_{1} \psi\left(z^{\prime}\right) f_{1}^{2}\left(z^{\prime}\right)+\alpha_{1} \psi\left(z^{\prime}\right)^{\left(a_{j}-2\right) / \alpha_{j}} f_{1}{ }^{0}\left(z^{\prime}\right)+\sum_{j>1} \alpha_{j}\left|z_{j}\right|^{a_{j}-2} f_{j}{ }^{1}\left(z^{\prime}\right)=0$

$(* *)_{6} \quad \alpha_{1} \psi\left(z^{\prime}\right) f_{1}^{1}\left(z^{\prime}\right)+\sum_{j>1} \alpha_{j}\left|z_{j}\right|^{a_{j}-2} \bar{z}_{j} f_{j}{ }^{0}\left(z^{\prime}\right)=i C\left(z^{\prime}\right)$

Concentrating our attention on the domain $D_{a}{ }^{0}: \psi\left(z^{\prime}\right)>0$, it follows from $(* *)_{5}$ that

$$
\left.\sum_{j>1} \alpha_{j}\left|z_{j}\right|^{a_{j}-2} z_{j} f_{j}^{1}\left(z^{\prime}\right)\right|_{\partial D^{0}{ }_{a}}=0
$$

to which Lemma 2.1 can be applied, and hence we conclude

$$
f_{2}^{1}(z)=\cdots=f_{n}^{1}(z)=0,
$$

which together with $(* *)_{5}$ implies that $f_{1}^{2}\left(z^{\prime}\right)=f_{1}^{0}\left(z^{\prime}\right)=0$. Similarly, it follows from $(* *)_{6}$

$$
\left.\operatorname{Re}\left(\sum_{j>1} \alpha_{j}\left|z_{j}\right|^{\alpha_{j}-2} \bar{z}_{j} f_{j}{ }^{0}\left(z^{\prime}\right)\right)\right|_{\partial D_{\gamma}{ }^{0}}=0
$$

to which our inductive hypothesis may be applied, and we have

$$
\begin{aligned}
& f_{j}{ }^{0}\left(z^{\prime}\right)=\frac{2}{\alpha_{j}}\left(i c_{j}-\sum_{k=r+1}^{n} \bar{c}_{k} z_{k}\right) z_{j} \quad(j=2,3, \cdots, r) \\
& f_{j}{ }^{0}\left(z^{\prime}\right)=i c_{j}+\sum_{k=r+1}^{n} c_{j k} z_{k}-\left(\sum_{k=r+1}^{n} c_{k} z_{k}\right) z_{j} \quad(j=r+1, \cdots, n) .
\end{aligned}
$$

Substituting these into $(* *)_{6}$, we finally have

so that

$$
\operatorname{Re}\left(\alpha_{1} f_{1}^{1}\left(z^{\prime}\right)-2 \sum_{k=r+1}^{n} \bar{c}_{k} z_{k}\right)=0
$$

$$
f_{1}^{1}\left(z^{\prime}\right)=\frac{2}{\alpha_{1}}\left(i c_{1}-\sum_{k=r+1}^{n} \bar{c}_{k} z_{k}\right)
$$

for some real number $c_{1}$.

Thus the Proposition 2.3. is proved. 
Remark. The set of all vector fields as are written in the Proposition 2.3 constitutes an infinitesimal transformation group of $D_{c}$ which we shall also denote by $\mathfrak{A}\left(\bar{D}_{c}\right)$, even if $\alpha_{1}, \cdots, \alpha_{n}$ are any positive real numbers. Actually every element of $\mathfrak{A}\left(\bar{D}_{\alpha}\right)$ is complete in $D_{a}$. To see this one may only note that

$X\left(\left|z_{1}\right|^{n_{1}}-\psi\left(z^{\prime}\right)\right) \equiv 0 \quad\left(\bmod .\left|z_{1}\right|^{n_{1}}-\psi\left(z^{\prime}\right)\right) X z_{j} \equiv 0 \quad\left(\bmod . z_{j}\right)(j=1, \cdots, r)$ for $X \in \mathfrak{A}\left(\bar{D}_{c s}\right)$. We denote the Lie transformation group which has $\mathfrak{U}\left(\bar{D}_{c}\right)$ as its infinitesimal group by $A\left(\bar{D}_{c}\right)$.

The rest of this Section we devote to investigate the structure of $\mathfrak{Y}\left(\bar{D}_{\kappa}\right)$, especially as a transformation group of $D_{n}$, and from now on we allow $\alpha_{1}, \cdots, \alpha_{n}$ to take any real numbers. To avoid inessential complications we only deal with the case where $\alpha_{1} \neq 2$, $\alpha_{2}=\cdots=\alpha_{n}=2$. Set

$$
\mathfrak{G l t}(n-1,1)=[A \in \mathcal{G} \ell(n, C): \operatorname{Sp}(A)=0, A \hat{I}+\hat{I} A=0]
$$

where $S p$ means the trace,

$$
\hat{I}=\left(\begin{array}{cccc}
-1 & & & \\
& 1 & & \\
& & \ddots & \\
& & & 1
\end{array}\right) .
$$

Clearly an element of $\mathfrak{s l t}(n-1,1)$ can be written as follows:

$$
A=\left(\begin{array}{l|l}
-S p(C) & \bar{c}_{2} \cdots \bar{c}_{n} \\
\hline c_{2} & C=\left(c_{j k}\right) \\
\vdots &
\end{array}\right)
$$

where $C=\left(c_{j k}\right)$ is an anti-Hermitian matrix. Now define for this $A$ an element $X(A)$ of $\mathfrak{U}\left(\bar{D}_{\alpha}\right)$ by setting

$$
\begin{aligned}
X(A)= & \operatorname{Re}\left(\frac{2}{\alpha_{1}}\left(S p(C)-\sum_{k>1} \bar{c}_{k} z_{k}\right) z_{1} \frac{\partial}{\partial z_{1}}\right. \\
& \left.+\sum_{j>1}\left(c_{j}+\sum_{k>1}\left(c_{j k} z_{k}+\delta_{j k} S p(C)\right) z_{k}-\left(\sum_{k>1} \bar{c}_{k} z_{k}\right) z_{j}\right) \frac{\partial}{\partial z_{j}}\right) .
\end{aligned}
$$

Then the mapping $A \rightarrow X(A)$ is a Lie algebra isomorphism onto a simple ideal of $\mathfrak{A}\left(\bar{D}_{c}\right)$, which shall be denoted by $\mathfrak{U}\left(\bar{D}_{\alpha}\right)$, and $\mathfrak{A}\left(\bar{D}_{\alpha}\right)$ is isomorphic to $\mathbb{R}+\mathfrak{g l t}(n-1,1)$ (the direct sum of Lie algebras).

We shall now investigate orbits of $\mathfrak{U}\left(\bar{D}_{c}\right)$ (i.e. maximal integral 
submanifold of $\left.\mathfrak{Y}\left(\bar{D}_{\alpha}\right)\right)$ and those of $\mathfrak{Y} *\left(\bar{D}_{c}\right)$. At first note that each element of $\mathfrak{A}\left(\bar{D}_{c}\right)$ is tangent to $D_{a}{ }^{\prime}=\left[z \in D_{a}: z_{1}=0\right]$, and that the restriction of $\mathfrak{Y}\left(\bar{D}_{\alpha}\right)$ to $D_{a}^{\prime}$ is transitive. Thus $D_{a}^{\prime}$ is an orbit of $\mathfrak{A}\left(\bar{D}_{c}\right)$. And it is also an orbit of $\mathfrak{A} *\left(\bar{D}_{c}\right)$. Direct inspection shows that $\operatorname{dim} \mathfrak{A}_{z}\left(\bar{D}_{\alpha}\right)=2 n-1$ if $z \in D_{\alpha}-D_{\alpha}{ }^{\prime}$, where $\mathfrak{A}_{z}\left(\bar{D}_{a}\right)=\left[X_{z}: X \in \mathfrak{A}\left(\bar{D}_{a}\right)\right]$. While a straightforward computation shows that

$$
X \log \left(1-\sum_{j>1}\left|z_{j}\right|^{\omega_{j}}\right)=X \log \left|z_{1}\right|^{\alpha_{1}} \quad \text { for } \quad X \in \mathfrak{A}\left(\bar{D}_{\alpha}\right) .
$$

From these it follows that the submanifold $M_{a}$ in $D_{a}$ defined by $\left|z_{1}\right|^{\infty}{ }^{\infty}=a\left(1-\sum_{j>1}\left|z_{j}\right|^{\infty}{ }_{j}\right) \quad(0 \leq a \leq 1)$ are orbits of $\mathfrak{U}\left(\bar{D}_{\infty}\right)$. Let $\mu$ be the projection of $D_{a}$ onto $D_{\alpha}^{\prime}$ which sends $\left(z_{1}, \cdots, z_{n}\right)$ to $\left(0, z_{2}, \cdots, z_{n}\right)$. Clearly $\mu$ maps orbits of $\mathfrak{U}\left(\bar{D}_{a}\right)$ onto $D_{a}^{\prime}$ and the intersection of each orbit of $\mathfrak{A}\left(\bar{D}_{a}\right)$ and $\mu^{-1}(K)$ is compact when $K$ is a compact subset of $D_{a}{ }^{\prime}$. Therefore $\mu$ also maps each orbit of $\mathfrak{U}^{*}\left(\bar{D}_{a}\right)$ onto $D_{a}{ }^{\prime}$. For, let $N$ be an orbit of $\mathfrak{Y} *\left(\bar{D}_{a}\right)$ and suppose that $z^{\prime} \in D_{a}^{\prime}$ belongs to $\partial \mu(N)$. Then $\mu^{-1}\left(z^{\prime}\right) \cap \partial N$ is non-empty since $\left.\mu\right|_{M_{a}}$ is proper as was seen above. Now choose $z^{0} \in \mu^{-1}\left(z^{\prime}\right) \cap \partial N$ and consider a sufficiently small neighbourhood of $z^{0}$ whose intersection with each orbit of $\mathfrak{A} *\left(\bar{D}_{a}\right)$ is mapped by $\mu$ to an open set of $D_{a}^{\prime}$ containing $z^{\prime}$. The existence of such a neighbourhood contradicts to $z^{0} \in \partial N$. Thus we have proved that $\left.\mu\right|_{N}$ is the covering mapping when $N$ is naturally topologized. While $D_{\alpha}^{\prime}$ is simply-connected, $\left.\mu\right|_{N}$ is a diffeomorphism.

Quite similar treatment is applicable also to the case where $\alpha_{1} \neq 2, \cdots, \alpha_{r} \neq 2, \alpha_{r+1}=\cdots=\alpha_{n}=2$. In this case $\mathfrak{A}\left(\bar{D}_{a}\right)$ is isomorphic to $\boldsymbol{R}^{I}+\mathfrak{S} \mathfrak{l t}(n-r, 1)$, the mapping $X: \mathfrak{g} \mathfrak{l}(n-r, 1) \rightarrow \mathfrak{A}\left(\bar{D}_{\alpha}\right)$ should be defined as follows :

$$
\begin{aligned}
X(A)= & \operatorname{Re}\left(\sum_{j=1}^{r} \frac{2}{\alpha_{j}}\left(S p(C)-\sum_{k>r} \bar{c}_{k} z_{k}\right) z_{j} \frac{\partial}{\partial z_{j}}\right. \\
& \left.+\sum_{j=r+1}\left(c_{j}+\sum_{k=r+1}^{n}\left(c_{j k}+\delta_{j k} S p(C)\right) z_{k}-\left(\sum_{j>k}^{n} \bar{c}_{k} z_{k}\right) z_{j}\right) \frac{\partial}{\partial z_{j}}\right)
\end{aligned}
$$

where $A=\left(\begin{array}{l|l}-S p(C) & c_{r+1} \cdots c_{n} \\ \hline c_{r+1} & C=\left(c_{j k}\right) \\ \vdots & \end{array}\right)$ 
The image of $X$ is denoted again by $\mathfrak{2} *\left(\bar{D}_{a}\right)$. In summary we have the following proposition.

Proposition 2. 4. Suppose that $\alpha_{1} \neq 2, \cdots, \alpha_{r} \neq 2, \alpha_{r+1}=\cdots=\alpha_{n}=2$. Then submanifolds of $D_{a}$ of dimension $\leq 2 n-r$ defined by $\left|z_{j}\right|^{\alpha}{ }_{j}=$ $a_{j}\left(1-\sum_{k>r}\left|z_{k}\right|^{a}{ }_{k}\right)\left(0<a_{j}<1, j=1,2, \cdots, r\right)$ are the orbits of $\mathfrak{A}\left(\bar{D}_{a}\right)$ and $\mu$ maps each orbit of $\mathfrak{U} *\left(\bar{D}_{a}\right)$ diffeomorphically onto $D_{a}$. Here $\mu$ is the projection which sends $\left(z_{1}, \cdots, z_{r}, z_{r+1}, \cdots, z_{n}\right)$ to $\left(0, \cdots, 0, z_{r+1}, \cdots, z_{n}\right)$ and $D_{\alpha}^{\prime}=\left[\left(0, \cdots, 0, z_{r+1}, \cdots, z_{n}\right) \in D_{\alpha}\right]$.

Next proposition is important in the next section.

Proposition 2.5. For each element $f$ of $A\left(\bar{D}_{a}\right)$ there exists one and only one element $\hat{f}$ of $A\left(\bar{D}_{a^{\prime}}\right)$ such that $\mu \cdot f=\hat{f} \cdot \mu$. Conversely, for each element $g$ of $A\left(\bar{D}_{a}^{\prime}\right)$ there corresponds one and only one $\widetilde{g}$ of $A *\left(\bar{D}_{a}\right)$ such that $\mu \cdot \widetilde{g}=g \cdot \mu$.

Proof. The former half of this follows immediately from the fact that

$$
\left[X, \frac{\partial}{\partial z_{j}}\right] \equiv 0 \quad\left(\bmod \cdot \frac{\partial}{\partial z_{1}}, \cdots, \frac{\partial}{\partial z_{r}}\right)
$$

for $X \in \mathfrak{U}\left(\bar{D}_{\alpha}\right)$.

The latter half follows from Proposition 2.4 as follows. At first observe that the mapping $f \rightarrow \hat{f}$ projects $A\left(\bar{D}_{\alpha}\right)$ onto $A\left(\bar{D}_{\alpha}^{\prime}\right)$ and that this mapping restricted to $A^{*}\left(\bar{D}_{a}\right)$ is a covering onto $A\left(\bar{D}_{a}\right)$. But it must be one to one since each orbit of $A^{*}\left(\bar{D}_{c}\right)$ is diffeomorphically mapped onto $D_{c}$ by $\mu$. Explicitly $g$ should be defined as follows:

$$
\widetilde{g}(z)=\left(\left.\mu\right|_{N_{z}}\right)^{-1}(g \mu(z))
$$

where $N_{z}$ is the orbit of $A^{*}\left(D_{a}\right)$ passing through $z$.

Remark. The identity component of $I\left(D_{a}\right)$ has been completely determined by the above consideration. For, this group consists of only linear elements by Lemma 1.2, and elements of its infinitesimal group are defined in the whole $\boldsymbol{C}^{n}$ and satisfy the same boundary condition as in the case $\alpha_{j} \equiv 0$ (mod. 2). Thus $I\left(D_{a}\right) \subseteq A\left(\bar{D}_{\alpha}\right)$, and we have

$$
I\left(D_{a}\right)=T \cdot U(n-r)
$$


where $U(n-r)$ denotes the unitary group for the last $n-r$ variables with respect to $\sum_{j=r+1}^{n}\left|z_{j}\right|^{2}$.

If $n=2, A^{0}\left(D_{a}\right)=A\left(\bar{D}_{a}\right)$ by Thullen [2]. So we would expect that $A^{0}\left(D_{a}\right)=A\left(\bar{D}_{a}\right)$. Fortunately this is true, which we shall show in the next section.

\section{Section 3}

This section we shall devote to the proof of Theorem B. In order to express the dependence on $n$ (the number of independent variables) of the statement of the Theorem $B$, we shall denote this by $S_{n}$, and we proceed by the induction on $n$. As was noted at the end of the Section 2, it suffices to show that $S_{n-1}$ implies $S_{n}$. Primarily we shall prove that $A^{0}\left(D_{\alpha}\right)=A\left(\bar{D}_{a}\right)$. As before rearranging the indices if necessary, we assume that $\alpha_{1} \neq 2, \cdots, \alpha_{r} \neq 2, \alpha_{r+1}=\cdots$ $=\alpha_{n}=2$. If $r=n$, there is nothing to prove by Corollary $\mathrm{A}$, therefore we assume that $r<n$.

Assertion 3.1. Any 1-parameter subgroup of $I\left(D_{a}\right)$ which has an analytic surface as its fixed point set is either $T_{j}(\xi)$ for some $j(1 \leq j \leq r)$, or $f^{-1} T_{r_{+1}}(\xi) f$ for some $f \in U(n-r)$. Further $T_{j}( \pm \xi)$ $(1 \leq j \leq r)$ is never conjugate to $T_{r+1}(\xi)$ by any element of $A\left(D_{a}\right)$.

The first statement of this assertion is almost evident if one note that for any 1-parameter subgroup $g_{t}$ of $I\left(D_{\infty}\right)$ there exists $f \in U(n-r)$ such that $f g_{t} f^{-1}$ is contained in $T$ since $T$ is a maximal torus of the compact group $I\left(D_{\alpha}\right)$. The second statement also immediately follows from the fact that $\left[z \in D_{\alpha}: z_{j}=0\right]$ (the fixed point set of $T_{j}(\xi)$ must not be holomorphically equivalent to $[z \in$ $\left.D_{a}: z_{r+1}=0\right]$ (the fixed point set of $T_{r+1}(\xi)$ ) by the assumption of induction.

To show $A^{0}\left(D_{c}\right)=A\left(\bar{D}_{a}\right)$, it suffices to prove that $O_{D_{a}}$ coincides with $D_{\alpha}{ }^{\prime}=\left[\left(0, \cdots, 0, z_{r+1}, \cdots, z_{n}\right) \in D_{a}\right]$ since isotropy subgroups of $A\left(\bar{D}_{\alpha}\right)$ and of $A^{0}\left(D_{c}\right)$ coincide. Suppose, conversely, that $z^{0}=g \cdot o \notin D_{\alpha}^{\prime}$ for some $g \in A^{0}\left(D_{c}\right)$. By Proposition 2.5 we may assume $z_{r+1}^{0}=\cdots$ $=z_{n}{ }^{0}=0$ since $A^{0}\left(\bar{D}_{a}{ }^{\prime}\right)$ operates transitively on $D_{a}{ }^{\prime}$. Then the 1- 
parametersubgroup $g^{-1} T_{r^{\perp_{1}}}(\xi) g$ leaves the origin fixed and hence belongs to $I\left(D_{a}\right)$. So $g^{-1} T_{r+1}(\xi) g=f T_{r+1}( \pm \xi) f^{-1}$ for some $f \in U(n-r)$ by Assertion 3.1. Therefore, if we replace $g$ by $g f$, we may assume $g^{-1} T_{r+1}(\xi) g=T_{r+1}( \pm \xi)$ and we still have $z^{0}=g \cdot o$. Thus $g$ maps $D_{\alpha}{ }^{0}=\left[z \in D_{a}: z_{r+1}=0\right]$ onto itself and hence $\left.g\right|_{D_{\alpha}{ }^{0}} \in A\left(D_{\alpha}{ }^{0}\right)$. But $A\left(\bar{D}_{a}^{0}\right)=A^{0}\left(D_{c}^{0}\right)$ by our assumption of induction.

Hence the orbit of $A\left(D_{a}{ }^{\circ}\right)$ passing through the origin must coincide with $\left[z \in D_{\infty}{ }^{0}: z_{1}=\cdots=z_{r}=0\right]$ since $A^{0}\left(D_{\infty}{ }^{0}\right)$ is a normal subgroup of $A\left(D_{a j}{ }^{0}\right)$ and since $\left[z \in D_{a j}{ }^{0}: z_{1}=\cdots=z_{r}=0\right]$ is the unique minimal orbit of $A^{0}\left(\bar{D}_{c}^{0}\right)$ by Proposition 2.5. Thus $z^{0}$ must be 0 , which contradicts to $z^{0} \notin D_{a}^{\prime}$. This proves that $A^{0}\left(D_{a}\right)=A\left(\bar{D}_{a}\right)$.

Now we shall prove the second half of $S_{n}$. Suppose that $D_{\alpha}, D_{\alpha^{\prime}}$ are mutually equivalent. Since $A^{0}\left(D_{\alpha}\right)=A^{0}\left(\bar{D}_{\alpha}\right)$, the minimal orbit of $A\left(D_{\alpha}\right)$ is unique. Then every holomorphic isomorphism of $D_{\alpha}$ onto $D_{\alpha^{\prime}}$ maps the unique minimal orbit of $A\left(D_{\alpha}\right)$ onto that of $A\left(D_{\alpha^{\prime}}\right)$. Each of these orbits contains the origin, so that there exists a holomorphic isomorphism which sends the origin into itself. Such an isomorphism is linear by Lemma 1.2. Thus $\alpha=\alpha^{\prime}$. Theorem B is completely proved.

\section{References}

[1] Behnke, H. and P. Thullen, Theorie der Funktionen mehrerer komplexer Veränderlichen, Springer, 1934.

[2] Borel, A., Kählerian coset spaces of semi-simple Lie groups, Proc. Nat. Acad. Sci. U. S. A. 40 (1954), 1147-1151.

[3] Cartan, E., Sur les domaines bornés homogènes de l'espace de $n$-variables complexes, Abh. Math. Sem. Hamburg, 11 (1935), 116-162.

[4] Cartan, H., Les fonctions de deux variables complexes et le problème de la représentation analytique, J. Math. Pures Appl. 10 (1931), 1-114.

[5] - Sur les transformations analytiques des domaines cerclés et semicerclés bornés, Math. Ann. 106 (1934), 540-573.

[6] Hano, J., On Kählerian homogeneous spaces of unimodular Lie groups, Amer. J. Math. 79 (1957), 885-900.

[7] Harish-Chandra, Representations of semi-simple Lie groups IV, Amer. J. Math. 77 (1955), 743-777.

[8] Koszul, J. L., Sur la forme Hermitienne canonique des especes homogènes complexes, Canad. J. Math. 7 (1955), 562-576.

[9] Kritikos, N., Über analytische Abbildungen einer Klasse von vierdimensionalen Gebieten, Math. Ann. 99 (1928), 321-341. 
[10] Piatetskii-Shapiro, I. I., Bounded homogeneous domains in $n$-dimensional space, Amer. Math. Soc. Transl. II-43 (1964).

[11] Reinhardt, K., Über Abbildungen durch analytische Funktionen zweier Veränderlichen, Math. Ann. 83 (1921), 211-255.

[12] Thullen, P., Zu den Abbildungen durch analytische Funktionen mehrerer Veränderlichen, Math. Ann. 104 (1931), 244-259; 373-376. 
